\title{
A compositional framework for energy management of a smart grid: a scalable stochastic hybrid model for cooling of a district network
}

\author{
Daniele Ioli, Alessandro Falsone, Simone Schuler, Maria Prandini
}

\begin{abstract}
The goal of this paper is to introduce a compositional modeling framework for the energy management of a smart grid that operates connected to the main grid. The focus is on the cooling of a district network composed of multiple buildings that possibly share resources such as storages, chillers, combined heat and power units, and renewable power generators. We adopt a modular perspective where components are described in terms of energy fluxes and interact by exchanging energy. Model dimension and complexity depend on the number and type of components that are present in the specific configuration. Energy management problems like the minimization of the electrical energy cost or the tracking of some electrical energy profile can be addressed in the proposed framework via different control strategies and architectures.
\end{abstract}

\section{INTRODUCTION}

In this paper we propose a compositional framework for modeling a smart grid to the purpose of efficient energy management. A smart grid is a subpart of the grid, with both, generators and loads, and, in principle, can be operated autonomously in island mode. Here, we consider the case when it is connected to the main grid and relies on it for balancing possible mismatches between electrical energy production and consumption. In this setting, stability is not a concern at the smart grid level, and energy management reduces to the task of satisfying the energy requests within the smart grid while optimizing the electrical energy exchanges with the main grid. If the goal is reducing the electrical energy cost, then, this can be achieved e.g. by using devices at their highest efficiency operating conditions, and shifting the energy request to the main grid to hours where prices are lower by means of storage systems and smart appliances. Smart grids can also contribute to ease the integration of renewable energy production and distributed energy generation in the main grid, since they can smooth out the high variability of the renewable energy production by implementing appropriate energy management strategies.

Motivated by the high consumption of electric energy in buildings (40\% of the overall electricity usage in US is due to buildings with almost a half of this fraction used for cooling, heating, and air conditioning, [1]), we shall focus on the cooling of a district network. The network is composed of multiple buildings possibly sharing resources such as storages, chillers, combined heat and power units, and renewable power generators like wind turbines. We adopt

This work is partially supported by the European Commission under the project UnCoVerCPS with grant number 643921.

Daniele Ioli, Alessandro Falsone, Maria Prandini are with Dipartimento di Elettronica, Informazione e Bioingegneria, Politecnico di Milano, Milano, Italy \{daniele.ioli, alessandro.falsone, maria.prandini\}@polimi.it

Simone Schuler is with the Controls and Embedded Systems Lab, GE Global Research, Munich, Germany simone.schuler@ge.com a modular perspective oriented to the energy management of the district. Different components are described in terms of thermal/electrical energy fluxes and interact by exchanging energy, with the overall balance of thermal and electrical energy in the district network equal to zero. Related work is described in [2], where the focus is only on modeling and control of a building. The proposed model for the building is bilinear and not easily integrable with that of other smart grid components.

Our modeling framework includes control inputs and stochastic disturbances. Inputs and state variables have both a discrete and a continuous component so that the overall model is a Stochastic Hybrid System (SHS), [3]. In this setting, one can address energy management problems like the minimization of the cost of the electrical energy requested to the main grid or the tracking of some given electrical energy exchange profile that was agreed with the main grid according to a demand-response strategy. The district network in the latter case can be viewed as a user that actively participates to the electrical energy demand/generation balance of the overall grid, and, hence, to its stabilization. Thermal storage systems can be particularly useful in this respect since they can serve the twofold purpose of making devices work close to their highest efficiency conditions, thus reducing the electrical energy consumption, and shifting in time the electrical energy request from the main grid, see e.g. [4], [5], [6], [7] for more details. Also the "building thermal mass" can be beneficially exploited as a passive thermal storage to add further flexibility to the system, [8], [9], [10].

\section{DISTRICT NETWORK COMPONENTS}

We adopt a modular and control perspective, oriented to the energy management of the district network along some finite time horizon $\left[t_{i}, t_{f}\right]$. To this purpose, we divide $\left[t_{i}, t_{f}\right]$ into $M$ time slots of duration $\delta_{t}$, and provide the contribution per time slot in terms of energy requested/provided or energy conversion of each component of the district network. Components can then be combined via energy balance equations. Some of the inputs are left free and can be set, e.g., to minimize the electrical energy costs.

\section{A. Building}

Consider a building composed of $n_{z}$ zones, each one with its own temperature profile $T_{z, j}, j=1, \ldots, n_{z}$, to track. We can then define the temperature profile of the building as $\boldsymbol{T}_{\boldsymbol{z}}=\left[T_{z, 1} \cdots T_{z, n_{z}}\right]^{\top}$. The building cooling energy demand for tracking $\boldsymbol{T}_{\boldsymbol{z}}$ along the discretized time horizon $\left[t_{i}, t_{f}\right]$ is given by the cooling energy request during the $k$-th time slot, namely $E_{c}(k)$, summed up over all the $M$ time slots: 
$E_{c}=\sum_{k=1}^{M} E_{c}(k)=\sum_{k=1}^{M} \sum_{j=1}^{n_{z}} E_{c, j}(k)$, where $E_{c, j}(k)$ is the energy request of zone $j$ during the $k$-th slot and is the sum of four contributions, namely

$$
E_{c, j}(k)=E_{w, j}(k)+E_{p, j}(k)+E_{\text {int }, j}(k)+E_{z, j}(k) .
$$

$E_{w, j}$ in (1) is the amount of energy exchanged between the walls and zone $j, E_{p, j}$ and $E_{\text {int }, j}$ is the heat produced respectively by people and by other sources of heat inside zone $j$, and $E_{z, j}$ is the energy contribution of the thermal inertia of zone $j$. We shall show next that the energy contributions in (1) are affine as a function of $\boldsymbol{T}_{\boldsymbol{z}}$. This is particularly convenient if $\boldsymbol{T}_{\boldsymbol{z}}$ is taken as control input to be set, e.g., for energy cost minimization.

1) Wall-zone energy exchange $E_{w}$ : The building is composed of zones that are separated through walls one from the other, and from the outside ambient as well. Each wall can be modeled as the composition of one-dimensional finite volumes by dividing it into vertical layers ('slices') that differ in width and material composition. The area of each slice coincides with the wall area and each slice is assumed to have a uniform density and a uniform temperature. The heat flow is perpendicular to the surface of the slice: each internal slice exchanges heat only with nearby slices through conduction, whilst boundary slices also exchange heat via convection and thermal radiation through surfaces that are exposed towards either a zone or the outside of the building. Based on the assumption that external surfaces are grey and opaque, with zero transmittance and equal absorbance and emissivity to shortwave and longwave radiation, in [10], the following expression for the thermal energy exchanged by the building structure to each thermal zone is derived: $\boldsymbol{E}_{\boldsymbol{w}}=$ $\tilde{F} x(0)+\tilde{G} \boldsymbol{u}+\tilde{H} \boldsymbol{w}$, where $\boldsymbol{E}_{\boldsymbol{w}}=\left[E_{w}^{\top}(1) \cdots E_{w}^{\top}(M)\right]^{\top}$, $x(0)$ is the initial condition of a suitably defined state vector, $\boldsymbol{u}=\left[u^{\top}(0) \cdots u^{\top}(M)\right]^{\top}$ is the input vector with $u(k)=$ $\boldsymbol{T}_{\boldsymbol{z}}\left(k \delta_{t}\right)$, and $\boldsymbol{W}=\left[\boldsymbol{W}_{\mathbf{1}}^{\top} \cdots \boldsymbol{W}_{\boldsymbol{n}_{\boldsymbol{w}}}^{\top}\right]^{\top}$ is the disturbance vector with $w(k)=\boldsymbol{d}\left(k \delta_{t}\right)$, where $\boldsymbol{d}=\left[T_{o} Q^{S} Q^{L} 1\right]^{\top}$ collects the outdoor temperature $T_{o}$, and the incoming shortwave $Q^{S}$ and longwave $Q^{L}$ radiations. The last entry in $\boldsymbol{d}$ is introduced to account for the presence of an affine term.

2) People energy contribution $E_{p}$ : Occupancy implies heat production and, in crowded places, it is a relevant contribution to heat generation. The heat power $Q_{p, j}$ produced by $n_{p, j}$ occupants in zone $j$ at temperature $T_{z, j}$ is obtained as the product of $n_{p, j}$ and a quadratic function of $T_{z, j}$ ([11]). Since such a function is almost linear in a sensible range of operating temperatures, it can be linearized around some comfort temperature $\bar{T}_{z, j}$, thus obtaining:

$$
Q_{p, j}=n_{p, j}\left(p_{1} T_{z, j}+p_{0}\right)
$$

where $p_{1}$ and $p_{2}$ are suitable coefficients. Since $T_{z, j}$ is assumed to be linear within each time slot, if also $n_{p, j}$ is approximated by a linear function of time as suggested in [7], then, equation (2) can be analytically integrated within $\left[(k-1) \delta_{t}, k \delta_{t}\right]$ to obtain the people energy contribution to the heating of zone $j: E_{p, j}(k)=q_{2, k} T_{z, j}\left(k \delta_{t}\right)+q_{1, k} T_{z, j}((k-$ 1) $\left.\delta_{t}\right)+q_{0, k}$, where the coefficients $q_{0, k}, q_{1, k}$, and $q_{2, k}$ are functions of $n_{p, j}$ at time $(k-1) \delta_{t}$ and $k \delta_{t}$ ([10]). The energy transferred to all zones in each time slot can be collected in $E_{p}(k)=\left[E_{p, 1}(k) \cdots E_{p, n_{z}}(k)\right]^{\top}$. Then, $\boldsymbol{E}_{\boldsymbol{p}}=$
$\left[E_{p}^{\top}(1) \cdots E_{p}^{\top}(M)\right]^{\top}$ is given by $\boldsymbol{E}_{\boldsymbol{p}}=N\left(n_{p}\right) \boldsymbol{u}+e\left(n_{p}\right)$, where $N\left(n_{p}\right)$ and $e\left(n_{p}\right)$ depend on the coefficients in (2) and $n_{p}$ is a vector of the occupancies in all zones of the building along the whole time horizon.

3) Other internal energy contributions $E_{\mathrm{int}}$ : There are many other types of heat sources that may affect the internal energy of a building, e.g. internal lighting, electrical equipment, daylight radiation through windows, etc. The overall heat flow rate transferred to zone $j$ can be expressed as

$$
Q_{\text {int }, j}(t)=\alpha_{j}(t) Q^{S}(t)+\kappa_{j} I_{\mathbb{R}_{+}}\left(n_{p, j}(t)\right)+\lambda_{j},
$$

where $\alpha_{j}(t)$ is a coefficient that takes into account the mean absorbance coefficient of zone $j$, the transmittance coefficients of the windows and their areas, sun view and shading factors, and radiation incidence angle. The thermal energy contribution to zone $j$ due to internal lightening and electrical equipment is composed of two contributions: a constant term $\lambda_{j}$, and an additional therm $\kappa_{j}$ that represents the change in internal lightening and electrical equipment when people are present. $I_{\mathbb{R}_{+}}(\cdot)$ denote the indicator function on the positive real values. Note that $Q_{\text {int }, j}$ does not depend on the longwave radiation because windows are usually shielded against it. (3) can be discretized and integrated in order to obtain the energy $E_{\text {int, } j}(k)$ during the $k$-th slot. We can collect the thermal energies of the zones in a vector $E_{\text {int }}(k)=\left[E_{\text {int }, 1}(k) \cdots E_{\text {int, } n_{z}}(k)\right]^{\top}$, and, finally, define $\boldsymbol{E}_{\text {int }}=\left[E_{\text {int }}^{\top}(1) \cdots E_{\text {int }}^{\top}(M)\right]^{\top}$.

4) Zones energy contributions $E_{z}$ : Observe that in order to lower the temperature of a zone we need to draw energy from the zone itself. This contribution to the overall thermal energy (1) in the building can be expressed as

$$
E_{z, j}(k)=-C_{z, j}\left(T_{z, j}\left(k \delta_{t}\right)-T_{z, j}\left((k-1) \delta_{t}\right)\right),
$$

where $C_{z, j}$ is the equivalent heat capacity of the $j$-th zone. To account for all time slots and all zones in the building, we can define $\boldsymbol{E}_{\boldsymbol{z}}=\left[E_{z}^{\top}(1) \cdots E_{z}^{\top}(M)\right]^{\top}$ where $E_{z}(k)=$ $\left[E_{z, 1}(k) \cdots E_{z, n_{z}}(k)\right]^{\top}$ and derive $\boldsymbol{E}_{\boldsymbol{z}}=Z \boldsymbol{u}+z$ from (4), where $Z$ and $z$ are suitably defined matrix and vector.

\section{B. Chiller plant}

A chiller plant converts electrical energy into cooling energy, which is then transferred to the building via, e.g., the chilled water circuit. Chillers can be modeled through:

$E_{c h, \ell}=\frac{a_{1} T_{o} T_{c w} \delta_{t}+a_{2}\left(T_{o}-T_{c w}\right) \delta_{t}+a_{4} T_{o} E_{c h, c}}{T_{c w}-\frac{a_{3}}{\delta_{t}} E_{c h, c}}-E_{c h, c}$,

where $E_{c h, \ell}$ is the electrical energy absorbed by the chiller in order to provide the cooling energy $E_{c h, c}$ in a time slot of duration $\delta_{t}$. Note that $E_{c h, \ell}$ depends also on the outdoor temperature $T_{o}$ and the temperature of the cooling water $T_{c w}$. The latter is typically regulated by low level controllers so that it is maintained almost at some prescribed optimal operational value. The chiller description (5) is derived from the original $\mathrm{Ng}$-Gordon model [12] which is based on entropy and energy balance equations. Coefficients $a_{1}$, $a_{2}, a_{3}, a_{4}$ characterize the chiller performance. Depending on their values, we can have different efficiency curves as given by the so-called Coefficient Of Performance (COP), i.e., the ratio between the produced cooling energy and the corresponding electrical energy consumption. 
1) Chiller approximation: A convex biquadratic approximation $E_{c h, \ell}=c_{1}\left(T_{o}\right)$ Eeps $_{c h, c}^{4}+c_{2}\left(T_{o}\right) E_{c h, c}^{2}+c_{3}\left(T_{o}\right)$ of the nonlinear $\mathrm{Ng}$-Gordon model (5) can be derived by using weighted least square to best fit the most relevant points, i.e, those that correspond to zero energy request and to the maximum COP values. Another possible convex approximation of (5) is via a PieceWise Affine (PWA) function. In this case, the nonlinear characteristic is approximated by the convex envelope of a finite number of affine terms $E_{c h, \ell}(k)=$ $\max \left\{m_{c}\left(T_{o}\right) E_{c h, c}(k)+q_{c}\left(T_{o}\right)\right\}$, where the coefficients of the affine terms are collected in vectors $m_{c}\left(T_{o}\right)$ and $q_{c}\left(T_{o}\right)$, and the max operator is applied componentwise.

2) On-off switching: The chiller absorbs some amount of electrical energy even when no cooling energy is produced. In order to have the possibility of switching it on and off, we introduce the binary variable $\delta_{c h}(k), k=1, \ldots, M$, that represents the on $\left(\delta_{c h}(k)=1\right)$ and off $\left(\delta_{c h}(k)=0\right)$ status of the chiller at time $k, k=0, \ldots, M$. The cooling energy request $E_{c h, c}(k)$ and on/off command $\delta_{c h}(k)$ are related via the logical condition

$$
\delta_{c h}(k)=1 \Leftrightarrow E_{c h, c}(k)>0 .
$$

Let $E$ be an upper bound on $u_{c h}$ and $\varepsilon$ a small quantity, typically set equal to the machine precision. Using the Conjunctive Normal Form in [13], (6) can be expressed as a mixed integer linear condition: $\varepsilon-\varepsilon\left(1-\delta_{c h}(k)\right) \leq$ $E_{c h, c}(k) \leq E \delta_{c h}(k)$, which leads to $\delta_{c h}(k)=0 \Leftrightarrow$ $E_{c h, c}(k)=0$ and $\delta_{c h}(k)=1 \Leftrightarrow E_{c h, c}(k) \in[\varepsilon, E)$, that are practically equivalent to (6).

We can rewrite the model of the chiller integrating the on-off condition as

$E_{c h, \ell}(k)=\left\{\begin{array}{l}\left(c_{1}\left(T_{o}\right) E_{c h, c}^{4}+c_{2}\left(T_{o}\right) E_{c h, c}^{2}+c_{3}\left(T_{o}\right)\right) \delta_{c h}(k) \\ \max \left\{m_{c}\left(T_{o}\right) E_{c h, c}(k)+q_{c}\left(T_{o}\right)\right\} \delta_{c h}(k),\end{array}\right.$

depending on the adopted approximation. The PWA formulation is particularly convenient since the product between a (piecewise) affine function $M x+q$ and a discrete variable $\delta$ can be reduced to a mixed integer linear condition [13].

\section{Combined heat and power unit: Microturbine}

A Combined Heat and Power (CHP) unit is a device that jointly produces electricity and heat power while consuming primal energy (i.e. fossil fuels or hydrogen) with the purpose of reducing the amount of energy wasted in the environment. In most cases one of these two products is a byproduct. For example, modern power plants recover scattered heat and deliver it for district heating purposes. Big sized CHPs are becoming widely used and highly performing. At the same time a large number of micro-CHP solutions are being developed, the most promising ones being microturbines and fuel cells that convert gas or hydrogen into heat and electricity. Combined Cooling, Heat and Power (CCHP) devices are also available that convert part of the produced heat into cooling energy. Here we consider a microturbine modeled through two static characteristics describing the electrical power production and the heat production, both as a function of the fuel volumetric flow rate. Figure 1 represents the characteristics of the C30 microturbine produced by Capstone company [14]. We can see that both curves are

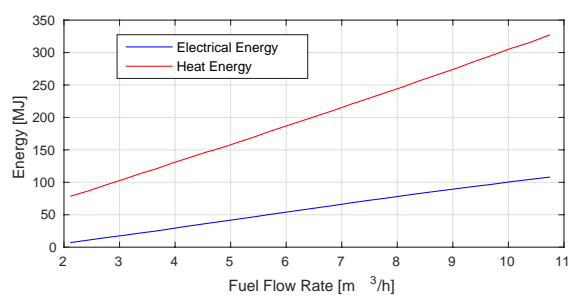

Fig. 1. Characteristic curves of the C30 microturbine.

almost linear. The electrical energy $E_{m t, \ell}(k)$ and the heat $E_{m t, h}(k)$ produced by this microturbine during the $k$-th time slot can then be expressed as affine functions of the fuel volumetric flow rate $u_{m t}(k)$, that is supposed to be constant in each time slot, i.e., $E_{m t, \ell}(k)=m_{\ell} u_{m t}(k)+q_{\ell}$, $E_{m t, h}(k)=m_{h} u_{m t}(k)+q_{h}$, where $m_{\ell}, q_{\ell}, m_{h}$, and $q_{h}$ are positive coefficients.

The microturbine specifications require a minimum fuel volumetric flow rate $u_{m t}^{\min }$ for the unit to be operative. We can introduce the possibility to switch the unit on or off. This is modeled by the binary variable $\delta_{m t}$ with the following logical condition associated: $u_{m t}(k) \leq u_{m t}^{\min } \Leftrightarrow \delta_{m t}(k)=0$. Such condition can be rewritten as: $\delta_{m t}(k)\left(u_{m t}^{\min }+\varepsilon\right) \leq$ $u_{m t}(k) \leq \delta_{m t}(k) u_{m t}^{\max }+\left(1-\delta_{m t}(k)\right) u_{m t}^{\min }$, where $u_{m t}^{\max }$ is the maximum flow rate and $\varepsilon>0$ is set equal to the machine precision. The microturbine model thus becomes $E_{m t, \ell}(k)=\delta_{m t}(k)\left(m_{\ell} u_{m t}(k)+q_{\ell}\right)$ and $E_{m t, h}(k)=$ $\delta_{m t}(k)\left(m_{h} u_{m t}(k)+q_{h}\right)$. Note that here we do not model explicitly the microturbine transient from on to off. If its duration is smaller than the time slot length $\delta_{t}$, then, the static model is accurate. For control purposes, we have two inputs that can be set: the fuel volumetric flow rate $u_{m t}(k)$ and the on/off status of the microturbine $\delta_{m t}(k), k=1, \ldots, M$.

\section{Storage}

Thermal Energy Storages (TESs) are becoming widely used in medium size grids. TESs represent the most effective way, or even sometimes the only way, to take advantage of renewable energy sources. This is indeed the case for thermal solar energy and geothermal energy systems. In a smart grid context, they can be used as energy buffers for unbinding energy production from energy consumption. More specifically, in a district cooling scenario, a TES for cooling energy can shift the production of cooling energy to off-peak hours of electrical energy consumption, make chillers operate in high-efficiency conditions, and smooth peaks of electrical energy request with benefits both for power production and distribution network systems.

There are many different technical solutions to store thermal energy, the most widely used are indeed fluid tanks and phase changing materials based storages. From an energy oriented perspective we will make use of a black box model, derived based on system identification techniques, that uses the energy exchange (drowned or inserted) as input and the thermal energy stored as output. This way of modeling does not consider the way energy is stored or provided. The simplest one is a first order AutoRegressive eXogenous (ARX) system: $S(k+1)=a S(k)-s(k)$, where $S(k)$ is the amount of cooling energy stored and $s(k)$ is the cooling energy exchanged $(s(k)>0$ if the storage is discharged, 
and $s(k)<0$ if it is charged), in the $k$-th time slot, while $a \in(0,1)$ is a coefficient introduced to model energy losses. Based on this model we can reformulate the thermal storage dynamics in a compact form as $\boldsymbol{S}=\Xi_{0} S(0)+\Xi_{1} s$, where we set $\boldsymbol{S}=[S(1) \cdots S(M)]^{\top}, \boldsymbol{s}=[s(0) \cdots s(M-1)]^{\top}$, and $\Xi_{0}$ and $\Xi_{1}$ are suitable matrices. Note that batteries for electrical energy can be modeled in the same way.

\section{E. Renewable energy generator: Wind turbine}

A wind turbine is used to convert the kinetic energy of air mass in motion to electrical energy. The maximum power that can be theoretically extracted from the wind is given by $P=\frac{1}{2} c_{p} \rho A v^{3}$, where $c_{p}$ is the power coefficient, $\rho$ is the air density, $A$ is the area covered by the rotor and $v$ is the wind speed. Wind power is thus proportional to the area covered by the rotor blades and proportional to the third power of the wind speed (see also Figure 2, dashed line). Thus, there are two possibilities to extract more power from the wind, either using larger turbine rotors, or installing the turbines at places with higher average wind speed. A simplistic nonlinear model for a wind turbine considers drive train shaft dynamics, tower fore-aft motion, and blade pitch dynamics (see, e.g., [15]). The wind turbine dynamics are highly nonlinear functions of the operating point defined by the wind conditions. These nonlinearities are described using the aerodynamic power and thrust coefficients. To the purpose of the energy management of the district network, we can consider a static model for the power produced by the wind turbine as a function of the wind speed as in Figure 2 (solid line). This figure shows that the actual power produced by the turbine is limited to some threshold value (the rated power) when a certain wind speed value is exceeded. Three different operational modes are typically defined: Region 1, where the wind speed is too low and the turbine is turned off; Region 2, below the rated power, where the power captured from the wind is maximized; Region 3, above the rated power, where the turbine blades are pitched out off the wind so that the power production is maintained constant and the loads on the turbine are minimized. The transition region between below-rated and above rated is called at-rated (region 2.5) and is marked via a circle in Figure 2. A turbine that is optimally sized for the site where it is installed is operating most of the time around this region. The wind acting on the rotor blades can therefore either be considered as a reference signal (Region 2) or a disturbance (Region 3). The conventional approach for controlling wind power production relies on the design of two control systems (a generator-torque controller and a full-span rotor-collective blade-pitch controller) that work independently, in the belowrated and above-rated wind-speed ranges, respectively ([16]).

\section{DISTRICT NETWORK CONFIGURATIONS}

In this section, we show how to compose the models previously introduced in order to define a scalable district network. Model composition is indeed easy given that each component is described in terms of thermal or electrical energy received as input and possibly provided as output. Energy balance equations and energy conversion functions can then be adopted to combine multiple network components. For instance, the sum of the cooling energy requests

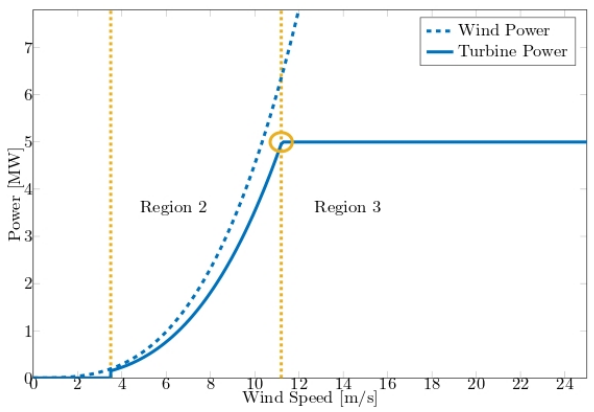

Fig. 2. Characteristic curve of the power production by a wind turbine.

of the buildings should be equal to the sum of the cooling energy provided by chillers and taken from/stored in the thermal storages; each chiller receives as input a cooling energy request and provides as output the corresponding electrical energy consumption; the sum of the electrical energy consumptions should be equal to the electrical energy produced by the local power generators, the CHP units, taken from/stored in the batteries, and provided by the main grid. Depending on the adopted model for each component, the overall model of the district network has a different complexity, the most general one being hybrid due to the presence of both continuous and discrete variables and stochastic due to the disturbances (e.g., occupancy, outside temperature, solar radiation, wind velocity) acting on the system.

Figure 3 shows a possible district network configuration and the energy fluxes between its components and the main grid. The district network is composed by multiple buildings that share common resources such as cooling and heat storages, chillers, CHP units, batteries and renewable energy generators. The three nodes appearing in the figure do not correspond to any physical component but are introduced to point out that fluxes associated with the same kind of energy (electrical, heat, and cooling energy) add up to zero. Some energy contributions can be controlled (e.g., those related to storage units), some others can be controlled only indirectly (e.g., electrical energy requested by the chiller), or cannot be controlled (e.g., renewable energy production). This is

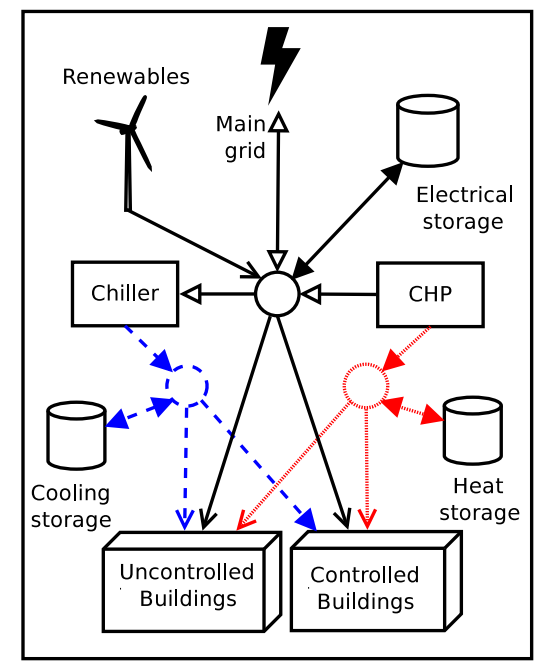

Fig. 3. District network configuration. The line style encodes the kind of energy: black thick, red thin, and blue dotted for electrical, heating, and cooling energy, respectively. Different arrowheads are used for energy fluxes that can be controlled, controlled only indirectly, or not controlled. 
pointed out using different arrowheads in Figure 3. As for buildings, some of them are controlled in that their cooling energy request can be modulated to some extent via the zone temperature set-point. If the zone temperature set-points are fixed and given by some comfort profiles, then the building is uncontrollable.

The electrical energy exchanged with the main grid is given by the amount of energy needed to maintain the balance between electrical energy demand and generation. A micro-grid becomes "smart" when it is possible to appropriately set the controllable variables so as to optimize its behavior. A sensible goal is to minimize the costs while guaranteeing the satisfaction of the energy needs of the users. Costs are mainly due to the electrical energy exchange with the main grid and by additional costs related to device operation such as startup and fuel costs. Startup costs may be also introduced to avoid continuous and unrealistic switchings of devices. The overall cost is then given by:

$$
J=C_{\ell}+C_{c h}+C_{m t}+C_{f}
$$

where the first term is the electrical energy cost $C_{\ell}=$ $\sum_{k=1}^{M} C_{\ell}(k) ; C_{c h}=\sum_{k=1}^{M} C_{c h}(k)$ is the cost for the chillers startup; $C_{m t}=\sum_{k=1}^{M} C_{m t}(k)$ and $C_{f}=\sum_{k=1}^{M} C_{f}(k)$ are the costs for the CHPs startup and fuel consumption.

Note that the cost formulation may involve introducing logical constraints. For example, a chiller startup cost can be modeled as $C_{c h}^{\xi} \max \left\{\delta_{c h}(k)-\delta_{c h}(k-1), 0\right\}$, where $C_{c h}^{\xi}$ is the actual startup cost which is accounted for at $k$ only if the chiller was off at $k-1$ and is switched on at $k$. Similarly, for the CHP, its startup cost at $k$ is given by $C_{m t}^{\xi} \max \left\{\delta_{m t}(k)-\right.$ $\left.\delta_{m t}(k-1), 0\right\}$. The fuel costs of a CHP are proportional to the amount of fuel consumption during the $k$-th time slot, i.e., $\psi_{f} \delta_{m t}(k) u_{m t}(k) \delta_{t}$, where $\psi_{f}$ is the unitary fuel cost. As for the electrical energy cost, the cost per time slot $C_{\ell}(k)$ is typically given by a PWA function of the electrical energy exchange $E_{L}(k)$ with the main grid, i.e.,

$$
C_{\ell}(k)=\max \left\{c_{1, \ell}(k) E_{L}(k)+c_{0, \ell}(k)\right\},
$$

where the coefficients of the affine terms are collected in vectors $c_{1, \ell}(k)$ and $c_{0, \ell}(k)$, and the max operator is applied componentwise. This expression allows to adopt different values for revenues $\left(E_{L}(k)<0\right)$ and actual costs $\left(E_{L}(k)>\right.$ $0)$, and to account for penalties when the electrical energy consumption/production $E_{L}(k)$ exceeds certain thresholds.

To describe $E_{L}$ for an arbitrary configuration, we use the following short-hand notations. Components correspond to energy contributions and are defined through letters (building $\mathcal{B}$, chiller $\mathcal{C}$, storage $\mathcal{S}$, CHP microturbine $\mathcal{M}$, wind turbine $\mathcal{W}$ ) with a superscript that denotes the model type (symbols are given in Table I) and the kind of energy (electrical $\ell$, cooling $c$, and heating $h$ ) provided as output. This is important in the case when a component allows for multiple kinds of energy as output. For instance, $\mathcal{M}^{B, h}$ stands for the heating energy produced by a CHP described by a linear on-off model. The subscript possibly denotes the energy request received as input. The electrical energy $E_{L}$ for the configuration in Figure 3 is then given by $E_{L}=$ $\mathcal{C}_{\leftarrow\left\{\mathcal{B}^{B, c}+\mathcal{B}^{A, c}+\mathcal{S}^{c}\right\}}^{A, \ell}+\mathcal{M}^{B, \ell}+\mathcal{S}^{\ell}+\mathcal{W}^{\ell}$. If we plug it into equation (8) and (7), we get the expression for the cost function $J$ to be minimized. Note that, in general, $J$ is uncertain in that it depends on the disturbances acting on the system, which means that either a min-max or an average cost criterion can be formulated if disturbances are explicitly accounted for. Furthermore, when we compose a district network model plugging together all the elements, we also get a number of constraints associated with them. Constraints express both technical limits (i.e., maximum cooling energy that a chiller can provide) and performance requirements (i.e., comfort temperature range). Additional constraints can be added if needed (e.g., the maximum amount of electrical energy that the main grid can provide). Yet, constraints might be uncertain due to the presence of disturbances, and, hence they might be enforced robustly or in probability.

Different formulations of the optimal energy management problem (certainty equivalence based, robust, stochastic) can then be considered for the minimization of the (nominal, min-max, average) cost in presence of (nominal, robust, probabilistic) constraints. Furthermore, different architectures (centralized, decentralized or distributed) can be conceived and implemented for the resulting optimization problem solution, depending on the actual communication and computation capabilities available in the network, and possible constraints dictated by privacy of information like each single building consumption and/or usage of local resources. Obviously, the dimension and complexity of the model depends on the specific configuration of the district network, and high dimensional and highly complex models might be a challenge for the application of certain control design strategies and architectures, thus calling for heuristic -though sensible- approaches, separating the discrete input optimization from the continuous input optimization (see, e.g., [17], [18]). Depending on the components that are introduced and their description, the resulting SHS model simplifies to a continuous system with continuous state and input variables, convex in the (continuous) control input, or it is hybrid with state and input that have both a continuous and a discrete component but with a dynamics that is affine in the continuous control input for each discrete mode. In the former case, convex programming can be adopted for optimal energy management [10]. In the latter case, a mixed integer linear programming formulation can be adopted [18].

\section{REFERENCES}

[1] D\&R International, Ltd., 2011 Buildings energy data book. U.S. Dep. of Energy, February 2012, http://buildingsdatabook.eren.doe.gov/.

[2] D. Sturzenegger, D. Gyalistras, V. Semeraro, M. Morari, and R. S. Smith, "BRCM Matlab Toolbox: Model generation for model predictive building control," in ACC, June 2014, pp. 1063-1069.

[3] J. Lygeros and M. Prandini, "Stochastic hybrid systems: a powerful framework for complex, large scale applications," European Journal of Control, vol. 16, no. 6, pp. 583-594, 2010.

[4] K. Deng, Y. Sun, A. Chakraborty, Y. Lu, J. Brouwer, and P. G. Mehta, "Optimal scheduling of chiller plant with thermal energy storage using mixed integer linear programming," in ACC, Washington, USA, 2013.

[5] K. M. Powell, W. J. Cole, U. F. Ekariaka, and T. F. Edgar, "Dynamic optimization of a campus cooling system with thermal storage," in ECC, Zurich, Switzerland, July 17-19 2013, pp. 4077-4082.

[6] Y. Ma, F. Borrelli, B. Hencey, A. Packard, and S. Bortoff, "Model predictive control of thermal energy storage in building cooling systems," in $C D C / E C C$, Dec 2009, pp. 392-397.

[7] F. Borghesan, R. Vignali, L. Piroddi, and M.Prandini, "Approximate dynamic programming-based control of a building cooling system with thermal storage," in IEEE ISGT 2013, Copenhagen, Denmark, 2013. 


\begin{tabular}{|c|c|c|c|c|}
\hline \multirow{6}{*}{ 离 } & Type & Description & Model & Optimization Problem \\
\hline & $\begin{array}{l}\text { A } \\
\text { B } \\
\text { C } \\
\text { D }\end{array}$ & $\begin{array}{l}\text { Biquadratic approximation } \\
\text { Piecewise approximation } \\
\text { Biquadratic on-off } \\
\text { Piecewise on-off }\end{array}$ & $\begin{array}{c}E_{c h, \ell}=c_{1} E_{c h, c}^{4}+c_{2} E_{c h, c}^{2}+c_{3} \\
E_{c h, \ell}=\max \left\{m_{c} E_{c h, c}+q_{c}\right\} \\
E_{c h, \ell}=\left(c_{1} E_{c h, c}^{4}+c_{2} E_{c h, c}^{2}+c_{3}\right) \delta_{c h} \\
E_{c h, \ell}=\max \left\{m_{c} E_{c h, c}+q_{c}\right\} \delta_{c h}\end{array}$ & $\begin{array}{l}\text { Convex-NL } \\
\text { MILP } \\
\text { MINLP } \\
\text { MINLP* }\end{array}$ \\
\hline & Var & Type & Description & Domain \\
\hline & $\begin{array}{c}E_{c h, \ell} \\
E_{c h, c} \\
\delta_{c h}\end{array}$ & $\begin{array}{c}\text { Output } \\
\text { Control input } \\
\text { Control input }\end{array}$ & $\begin{array}{c}\text { Absorbed electrical energy } \\
\text { Cooling energy request } \\
\text { On-off logical status }\end{array}$ & $\begin{array}{c}\text { Real } \\
\text { Real } \\
\text { Binary }\end{array}$ \\
\hline & Type & Constraint & Inequalities & Var \\
\hline & $\begin{array}{l}\text { A-D } \\
\text { A-D } \\
\text { C-D }\end{array}$ & $\begin{array}{c}\text { Electrical energy bounds } \\
\text { Cooling energy bounds } \\
\text { Logical on-off }\end{array}$ & $\begin{array}{c}0 \leq E_{c h, \ell} \leq E_{c h, \ell}^{\max } \\
0 \leq E_{c h, c} \leq E_{c h, c}^{\max } \\
E_{c h}(k) \geq \varepsilon-\varepsilon\left(1-\delta_{c h}(k)\right) \\
\left.E_{c h}(k) \leq E \delta_{c h}(k)\right)\end{array}$ & $\begin{array}{c}\text { Output } \\
\text { Input } \\
\text { Input }\end{array}$ \\
\hline \multirow{6}{*}{$\overbrace{0}^{\infty}$} & Type & Description & Model & Optimization Problem \\
\hline & $\begin{array}{l}\text { A } \\
\text { B }\end{array}$ & $\begin{array}{l}\text { Linear controllable } \\
\text { Linear simulator }\end{array}$ & $\begin{array}{l}E_{c}=B T_{z}+W d+b \\
E_{c}=B \bar{T}_{z}+W d+b\end{array}$ & $\begin{array}{c}\mathrm{LP} \\
/\end{array}$ \\
\hline & Var & Type & Description & Domain \\
\hline & $\begin{array}{c}T_{z} \\
d \\
E_{c} \\
E_{c, j}\end{array}$ & $\begin{array}{l}\text { Control input } \\
\text { Uncontrollable input } \\
\text { Output } \\
\text { Output }\end{array}$ & $\begin{array}{l}\text { Zone temperatures } \\
\text { Disturbances } \\
\text { Cooling energy request by building } \\
\text { Cooling energy request by zone } j\end{array}$ & $\begin{array}{l}\text { Real } \\
\text { Real } \\
\text { Real } \\
\text { Real }\end{array}$ \\
\hline & Type & Constraint & Inequalities & Var \\
\hline & A & $\begin{array}{l}\text { Comfort bounds } \\
\text { Cooling energy bounds }\end{array}$ & $\begin{array}{c}T_{z}^{\min } \leq T_{z} \leq T_{z}^{\max } \\
0 \leq E_{c} \leq E_{c}^{\max } \\
0 \leq E_{c, j} \leq E_{c, j}^{\max }\end{array}$ & $\begin{array}{l}\text { Input } \\
\text { Output }\end{array}$ \\
\hline \multirow{6}{*}{ 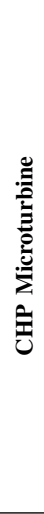 } & Type & Description & Model & Optimization Problem \\
\hline & A & $\begin{array}{l}\text { Linear } \\
\text { Linear on-off }\end{array}$ & $\begin{array}{c}E_{m t, \ell}(k)=m_{\ell} u_{m t}(k)+q_{\ell} \\
E_{m t, h}(k)=m_{h} u_{m t}(k)+q_{h} \\
E_{m t, \ell}(k)=\delta_{m t}(k)\left(m_{\ell} u_{m t}(k)+q_{\ell}\right) \\
E_{m t, h}(k)=\delta_{m t}(k)\left(m_{h} u_{m t}(k)+q_{h}\right)\end{array}$ & $\begin{array}{c}\text { LP } \\
\text { MINLP* }\end{array}$ \\
\hline & Var & Type & Description & Domain \\
\hline & $\begin{array}{c}E_{m t, \ell} \\
E_{m t, h} \\
u_{m t} \\
\delta_{m t} \\
\end{array}$ & $\begin{array}{c}\text { Output } \\
\text { Output } \\
\text { Control input } \\
\text { Control input }\end{array}$ & $\begin{array}{l}\text { Produced electricity } \\
\text { Produced heat } \\
\text { fuel inlet } \\
\text { On-off logical status }\end{array}$ & $\begin{array}{c}\text { Real } \\
\text { Real } \\
\text { Real } \\
\text { Binary } \\
\end{array}$ \\
\hline & Type & Constraint & Inequalities & Var \\
\hline & $\begin{array}{l}\text { A-B } \\
\text { C-D }\end{array}$ & $\begin{array}{l}\text { Fuel inlet bounds } \\
\text { Logical on-off }\end{array}$ & $\begin{array}{c}0 \leq u_{m t} \leq u_{m t}^{\max } \\
u_{m t}(k) \leq \delta_{m t}(k) u_{m t}^{\max }(k)+u_{m t}^{\min }(k) \\
u_{m t}(k) \geq \delta_{m t}(k)\left(u_{m t}^{\min }(k)+\varepsilon\right)\end{array}$ & $\begin{array}{l}\text { Output } \\
\text { Input }\end{array}$ \\
\hline \multirow{6}{*}{ 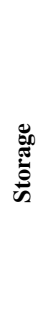 } & Type & Description & Model & Optimization Problem \\
\hline & A & Storage & $\boldsymbol{S}=\Xi_{0} S(0)+\Xi_{1} \boldsymbol{s}$ & 1 \\
\hline & Var & Type & Description & Domain \\
\hline & $\begin{array}{l}S \\
s\end{array}$ & $\begin{array}{c}\text { Output } \\
\text { Control input }\end{array}$ & $\begin{array}{l}\text { Energy content } \\
\text { Energy exchange }\end{array}$ & $\begin{array}{l}\text { Real } \\
\text { Real }\end{array}$ \\
\hline & Type & Constraint & Inequalities & Var \\
\hline & $\begin{array}{l}\mathrm{A} \\
\mathrm{A}\end{array}$ & $\begin{array}{l}\text { Energy content bounds } \\
\text { Energy exchange bounds }\end{array}$ & $\begin{array}{c}0 \leq \boldsymbol{S} \leq \boldsymbol{S}^{\max } \\
\boldsymbol{s}^{\min } \leq \boldsymbol{s} \leq \boldsymbol{s}^{\max }\end{array}$ & $\begin{array}{l}\text { Output } \\
\text { Input }\end{array}$ \\
\hline
\end{tabular}

TABLE I

[8] C. Balaras, "The role of the thermal mass on the cooling load of buildings. an overview on computational methods," Energy and Buildings, vol. 24, 1996.

[9] Y. Ma, A. Kelman, A. Daly, and F. Borrelli, "Predictive control for energy efficient buildings with thermal storage: Modeling, simulation and experiments," IEEE Control Systems Magazine, vol. 1, no. 1, pp. 45-66, 2012.

[10] D. Ioli, A. Falsone, and M. Prandini, "Optimal energy management of a building cooling system with thermal storage: A convex formulation," in ADCHEM, Whistler, British Columbia, Canada, June 2015.

[11] K. J. Butcher, CIBSE Guide A: Environmental Design. Norwich, UK: CIBSE Publications, 2006.

[12] M. Gordon and K. Ng, Cool thermodynamics. Cambridge International Science Publishing, 2000.

[13] A. Bemporad and M. Morari, "Control of systems integrating logic dynamics, and constraints," Automatica, vol. 35, no. 3, pp. 407-427,
1999.

[14] Capstone, Technical Reference Capstone Model C30 Performance. USA: Capstone, 2014

[15] D. Schlipf, D. J. Schlipf, and M. Kühn, "Nonlinear model predictive control of wind turbines using LIDAR," Wind Energy, vol. 16, no. 7, pp. 1107-1129, Oct. 2013.

[16] J. M. Jonkman, S. Butterfield, W. Musial, and G. Scott, "Definition of a 5-MW reference wind turbine for offshore system development," National Renewable Energy Laboratory, Tech. Rep. NREL/TP-50038060, 2009.

[17] S. R. Cominesi, M. Farina, L. Giulioni, B. Picasso, and R. Scattolini, "Two-layer predictive control of a micro-grid including stochastic energy sources," in ACC, Chicago, IL, USA, 2015, pp. 918-923.

[18] D. Ioli, A. Falsone, and M. Prandini, "An iterative scheme to hierarchically structured optimal energy management of a microgrid," in $C D C$, Osaka, Japan, December 2015. 